\title{
The Catalytic Centre of Transition Metal Chalcogenides vis-à-vis the Oxygen Reduction Reaction: An In Situ Electrochemical EXAFS Study
}

\author{
N. Alonso-Vante, M. Fieber-Erdmann*, H. Rossner*, E. Holub-Krappe*, Ch. Giorgetti**, \\ A. Tadjeddine**, E. Dartyge**, A. Fontaine** and R. Frahm***
}
Hahn-Meitner-Institut, Department Solare Energetik CS, Glienicker Str. 100, 14109 Berlin, Germany * Hahn-Meitner-Institut, PG Synchrotronstrahlung AS, Glienicker Str. 100, 14109 Berlin, Germany
** LURE, Bât. 209D, Centre Universitaire Paris-Sud, 91405 Orsay cedex, France *** HASYLAB at DESY, Notkestrasse 85, 22603 Hamburg, Germany

\begin{abstract}
The Chevrel type chaicogenide with the stoichiometry $\mathrm{Mo}_{6-\mathrm{x}} \mathrm{M}_{\mathrm{x}} \mathrm{X}_{8}$ (M=transition metal, e.g., $\mathrm{M}_{\mathrm{x}}=\mathrm{R} \mathrm{u}_{2}$; $\mathrm{X}=$ Chalcogene atom, e.g., S, Se) containing octahedral mixed-metal clusters of transition-metal atoms was investigated with respect to the oxygen reduction process in an in situ electrochemical enviroment via the EXAFS technique. The spectra analysis on $\mathrm{Ru}$ and $\mathrm{Mo}$ edges as a function of applied electrode potential revealed that ruthenium centers were preferentially modified by the presence of oxygen in the electrolyte: The variation in distance between $\mathrm{Ru}-(\mathrm{Ru}, \mathrm{Mo})$ was more sensitive in oxygen saturated solution as compared to $\mathrm{Mo}-(\mathrm{Ru}, \mathrm{Mo})$ and $\mathrm{Ru}-\mathrm{Se}$.
\end{abstract}

\section{INTRODUCTION}

The development of transition metal containing compounds for electrocatalysis with high activity and selectivity in acid medium led to the use of model system structures: Chevrel phase $\left(\mathrm{Mo}_{6-\mathrm{x}} \mathrm{M}_{\mathrm{x}} \mathrm{X}_{8}\right.$, where, e.g., $\mathrm{M}_{\mathrm{x}}=\mathrm{Ru}_{2}$ and $\left.\mathrm{X}=\mathrm{S}, \mathrm{Se}\right)$ [1-3]. The direct reduction of molecular oxygen to water (four-electrons) in acid medium reveals the capacity of such materials for the multi-electron charge transfer process, see figure 1 . Electrochemically speaking, the reactivity can be due to the structure of redox species of an interfacial complex formed during the charge transfer process. Therefore, it is of importance to determine the metal-metal-distance between atoms and their coordination during electrocatalysis. We report on in situ electrochemicalextended X-ray absorption fine structure (EXAFS) studies of the $\mathrm{Mo}_{4} \mathrm{Ru}_{2} \mathrm{Se}_{8}$ cluster under controlled electrode potential measured at the $\mathrm{Ru}$ and $\mathrm{Mo} \mathrm{K}$ - edges.

\section{EXPERIMENTAL}

Electrodes were prepared by rubbing a mixture of carbon paste and the cluster material onto porous carbon paper (1.3 $\mathrm{cm} \times 2$ $\mathrm{cm}$ ). The electrode with a thickness of ca. $0.4 \mathrm{~mm}$ was placed into an electrochemical cell with a three-electrode configuration and provided with Capton windows. The electrolyte used was $0.5 \mathrm{M} \mathrm{H}_{2} \mathrm{SO}_{4}$ (Merck). The solution was saturated with pure oxygen. A standard electrochemical arrangement with a three electrode cell was employed. Electrode potentials were controlled

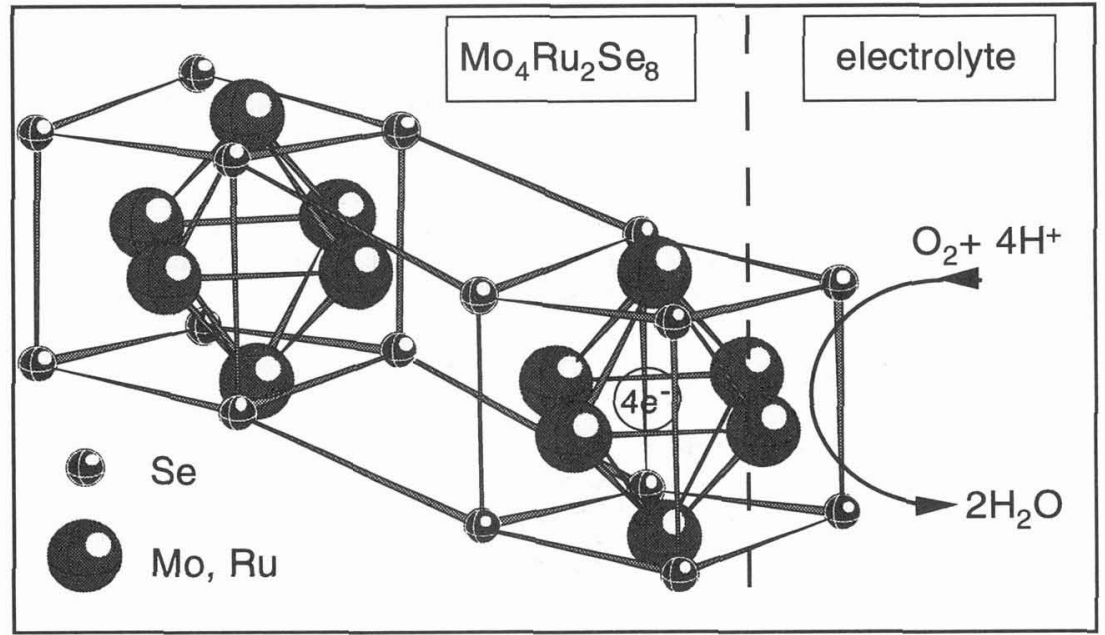

Figure 1: Interaction of molecular oxygen in acid medium at the Chevrel phase clusters - electrolyte interface. 


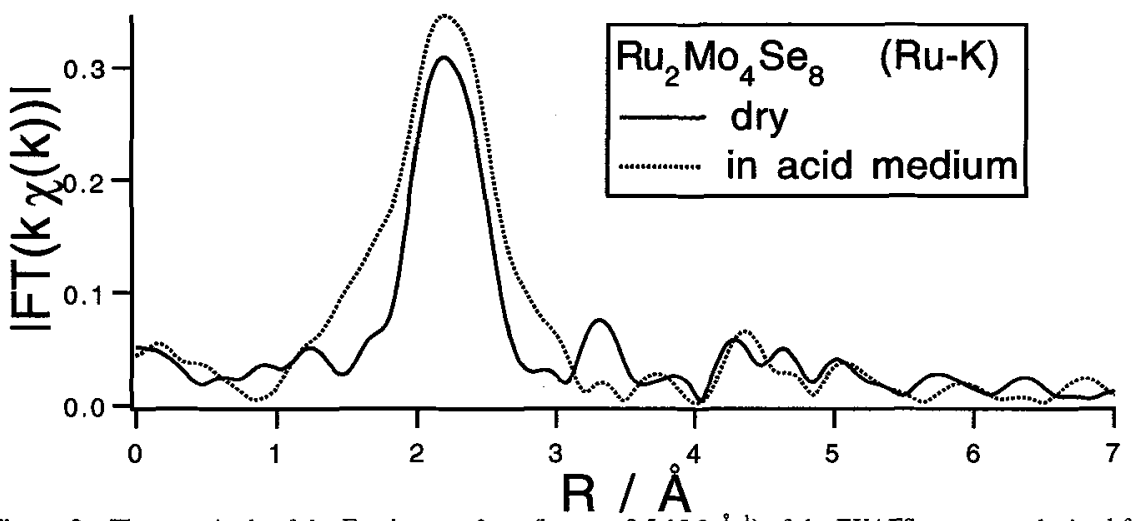

Figure 2a: The magnitude of the Fourier transform ( $k$ range $\left.3.5-15.8 \AA^{-1}\right)$ of the EXAFS spectrum obtained for the Ru K-shell.

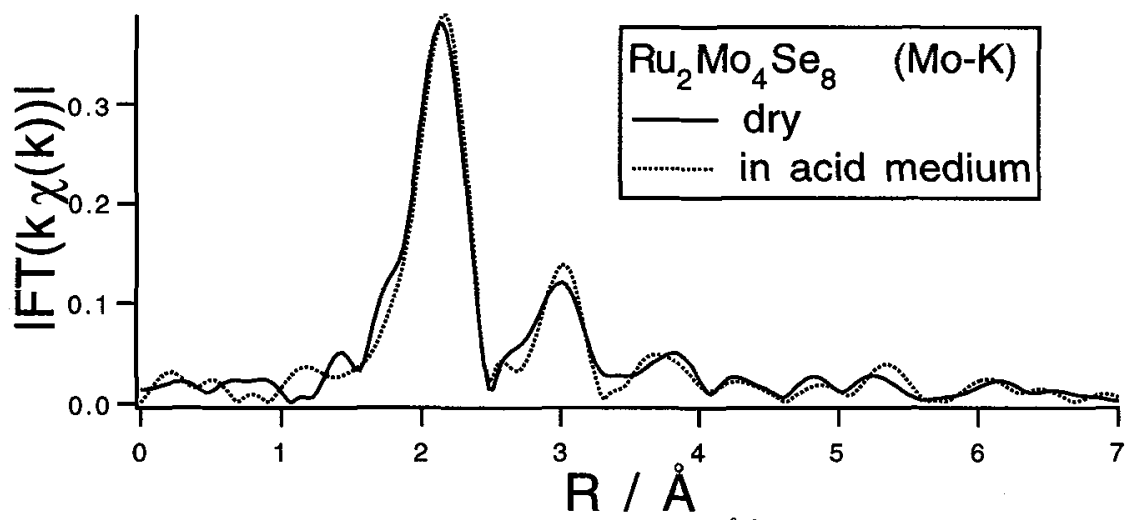

Figure $2 b$ : The magnitude of the Fourier transform (k range $3.5-15.8 \AA^{-1}$ ) of the EXAFS spectrum obtained for the Mo K-shell.

via a potentiostate PAR Model 273 interfaced by a PC. The counter electrode was a net of platinum placed in a separated compartment; Mercury sulphate electrode (MSE) in $0.5 \mathrm{M} \mathrm{H}_{2} \mathrm{SO}_{4}$ was used as a reference electrode (MSE=0.65 V/NHE). Prior to measurements the electrolyte was outgassed with argon and the electrode was activated by scanning at a constant rate, $50 \mathrm{mV} / \mathrm{s}$, between 0 and $-0.6 \mathrm{~V} / \mathrm{MSE}$. Thereafter, the EXAFS spectra were recorded at fixed electrode potentials in argon and with pure saturated oxygen electrolyte. Initial in situ experiments were peformed at the DCI dispersive line of LURE in transmission mode. Spectra were also recorded in transmission mode at the RÖMO II station at HAYSLAB. Prior to in situ measurements, spectra of dry samples were recorded in the cell. Thereafter, electrolyte was added and spectra were recorded as a function of the applied electrode potential. The EXAFS spectra were recorded at room temperature.

The Ramsauer-Townsend effect was taken into account [4] by substracting the background and normalizing the spectra to isolate the EXAFS modulation $\chi(k)$ as a function of the photoelectron wavenumber, $\mathrm{k}$, defined as: $\mathrm{k}=\left[2 \mathrm{~m}\left(\mathrm{E}-\mathrm{E}_{0}\right) / \mathrm{h}^{2}\right)^{1 / 2}$, where $E$ is the photon energy, $m$ is the electron mass, and $E_{0}$ the photoelectron energy whose value was initially chosen at the inflection point of the edge. The $\mathrm{Mo}_{4} \mathrm{Ru}_{2} \mathrm{Se}_{8}$ EXAFS spectra were first analyzed by the program FEFF 6.01 [5,6] based on an ab initio calculation using rhombohedral lattice as a model [7]. Then the program FEFFIT 3.21 [6] was used to extract the structure parameter such as $E_{0}$, the atomic distance $R$, the coordination numbers $N$ and the Debye-Waller factors exp(-2k $\left.{ }^{2} \sigma^{2}\right)$; $\sigma^{2}$ representing the mean square deviation from the average distance between the absorbing atom and the scattering atoms. For the analysis the data were Fourier transformed to $\mathrm{R}$ space by using $\mathrm{k}^{3}$ weightings and Gaussian window.

\section{RESULTS AND DISCUSSION}

Figure 2a shows the magnitude of the Fourier transform of the EXAFS spectrum of the Chevrel phase $\left(\mathrm{Mo}_{4} \mathrm{Ru}_{2} \mathrm{Se}_{8}\right)$ obtained for the $R u K$-shell in open circuit condition. The maximum peak is due to the $R u-S e(\vec{R}=2.47 \pm 0.05 \AA)$ and $R u-R u$ ( $\overline{\mathbf{R}}=2.73 \pm 0.05 \AA)$ distances, which were calculated with the phase shift obtained from FEFF. We observe that in electrolyte and under potentiostatic conditions this peak is higher and broader than that recorded in dry state. On the other hand, the magnitude of the Fourier transform of the Mo-K shell, see figure $2 \mathrm{~b}$, remains unchanged under similar conditions. This is in contrast to the Ruthenium edge. This qualitative comparison leads to the assumption that electrocatalysis of oxygen reduction 
takes place mainly at the ruthenium centres. More insight about this phenomenon is, however, obtained by visualizing the change in distances between the $\mathrm{Ru}$ and metal $(\mathrm{Ru}, \mathrm{Mo})$ or chalcogene (Se) as a function of the applied electrode potential in the presence of argon and oxygen, see Figure 3 . Within the limits of estimation error, the distance variation between Mo-(Mo-Ru) is not significant, whereas a significant distance change, attributed to $\mathrm{Ru}-\mathrm{Ru}$ induced by the presence of molecular oxygen, is observed. This result supports the observation already made in figure 2 . Interestingly, the distance change attributed to $\mathrm{Ru}$ and Se remains insensitive in the presence of argon or oxygen in the potential interval in which electrocatalysis takes place $(0.7 \mathrm{~V}>\mathrm{U}>0 \mathrm{~V})$, but its trend apparently follows in a parallel way the distance variation of $R u-R u$. This represents a strong indication of the cluster unit dynamics, cf. figure 1 , during electrocatalysis of the oxygen reduction and might be, to the best of our knowledge, a first in situ obeservation of such a process using this model system. Furthermore, the variation of coordination of oxygen with ruthenium as a function of the electrode potential is shown in the inset of figure 3 . The decrease of this value to negative bias potential suggests that the coordinated oxygen on the ruthenium transition metal is consumed and readily converted to water as reported earlier using the ring rotating disk electrode [8].

Due to the correlation between the Debye-Waller factor, $\sigma^{2}$, and the coordination number, $N$, it is not possible to estimate them separately, though both parameters describe the amplitude of $\chi(\mathbf{k})$. However, the fit procedure was only possible when one of these parameters was kept constant. The results show that at higher bias negative electrode potential, i.e., in which the electrochemical reaction is favoured, the magnitude of $\sigma^{2} \mathrm{Ru}-\mathrm{Ru}$ decreases, whereas the $\sigma^{2}{ }_{\mathrm{Ru}-\mathrm{Se}}$ increases. Regarding the structure of the crystal, the Ru-Se atomic pair are distorted during the reaction, while the $\mathrm{Ru}-\mathrm{Ru}$ atomic pair improves the geometry of the crystal structure.

Regardless of the complex structure of the analysed powder catalyst embedded in a carbon matrix, it was possible to detect a significant change of the material in a defined environment with the application of the EXAFS technique. This appraoch provided some clues about the dynamics of the cluster units during electrocatalysis.

\section{References}

[1] Alonso-Vante N. and Tributsch H., Nature 323 (1986) 431.

[2] Alonso-Vante N., Schubert B. and Tributsch H., Mat. Chem. Phys. 22 (1989) 281.

[3] Alonso-Vante N. and Tributsch H., Electrochemistry of Novel Materials- Vol. III, J. Lipkowski and P. N. Ross Eds. (VCH Publishers, Inc. USA, 1994) pp. 1-63.

[4] Rehr J.J., Booth C.H., Bridges F. and Zabinsky S.I., Phys. Rev. B 49(17) (1994) 12347.

[5] Rehr J.J., Mustre de Leon J., Zabinsky S.I. and Albers R.C., J. Am. Chem. Soc. 113 (1991) 5135.

[6] Newville M., Ravel B., Haskel D., Rehr J.J., Stern E.A. and Yacoby Y., Physica B 208 \& 209 (1995) 154.

[7] Hönle W., Flack H.D. and Yvon K., J. Sol. State Chem 49 (1983) 157.

[8] Alonso-Vante N., Jägermann W., Tributsch H., Hönle W. and Yvon K., J. Am. Chem. Soc. 109 (1987) 3251. 